\title{
K-NN supervised learning algorithm in the predictive analysis of the quality of the university administrative service in the virtual environment
}

\author{
Omar Freddy Chamorro Atalaya ${ }^{1}$, Guillermo Morales-Romero ${ }^{2}$, Adrián Quispe-Andía ${ }^{2}$, Beatriz \\ Caycho-Salas ${ }^{3}$, Elizabeth Katerin Auqui-Ramos ${ }^{2}$, Primitiva Ramos-Salazar ${ }^{4}$, Carlos Palacios-Huaraca $^{5}$ \\ ${ }^{1}$ Faculty of Engineering and Management, National Technological University of Lima Sur, Lima, Perú \\ ${ }^{2}$ Facultad de Ciencias, Universidad Nacional de Educación Enrique Guzmán y Valle, Lima, Perú \\ ${ }^{3}$ Facultad de Ciencias Empresariales, Universidad Nacional de Educación Enrique Guzmán y Valle, Lima, Perú \\ ${ }^{4}$ Facultad de Tecnología, Universidad Nacional de Educación Enrique Guzmán y Valle, Lima, Perú \\ ${ }^{5}$ Departamento de Ciencias, Universidad Tecnológica del Perú, Lima, Perú
}

\begin{tabular}{l} 
Article Info \\
\hline Article history: \\
Received Jul 25, 202 \\
Revised Oct 14, 2021 \\
Accepted Nov 26, 2021 \\
\hline Keywords: \\
K-nearest neighbor \\
Predictive analytics \\
Quality of service \\
Supervised learning \\
Virtuality
\end{tabular}

Article Info

Received Jul 25, 2021

Revised Oct 14, 2021

Accepted Nov 26, 2021

Virtuality

\begin{abstract}
The objective of this study is to analyze and discuss the metrics of the predictive model using the K-nearest neighbor (K-NN) learning algorithm, which will be applied to the data on the perception of engineering students on the quality of the virtual administrative service, such as part of the methodology was analyzed the indicators of accuracy, precision, sensitivity and specificity, from the obtaining of the confusion matrix and the receiver operational characteristic (ROC) curve. The collected data were validated through Cronbach's Alpha, finding consistency values higher than 0.9 , which allows to continue with the analysis. Through the predictive model through the Matlab R2021a software, it was concluded that the average metrics for all classes are optimal, presenting a precision of $92.77 \%$, sensitivity $86.62 \%$, and specificity $94.7 \%$; with a total accuracy of $85.5 \%$. In turn, the highest level of the area under the curve (AUC) is 0.98, which is why it is considered an optimal predictive model. Having carried out this study, it is possible to contribute significantly to the decision-making of the higher institution in relation to the improvement of the quality of the virtual administrative service.
\end{abstract}

This is an open access article under the CC BY-SA license.

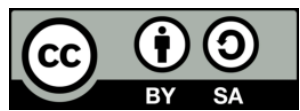

\section{Corresponding Author:}

Omar Freddy Chamorro Atalaya

Faculty of Engineering and Management, National Technological University of Lima Sur

Av. Central y Av. Bolivar, Sector 3, Grupo 1A-03, Villa El Salvador, Lima, Perú

Email: ochamorro@untels.edu.pe

\section{INTRODUCTION}

Today it is essential that the various organizations have the tools to capture, analyze and adapt to changes; even more so in this environment of high competition in institutions, the culture of organizational learning is considered key for decision-making based on the achievement of goals, to cement permanence in the market and to transcend it [1], [2]. In the virtual context in which we find ourselves, educational institutions apply techniques to understand the modeling of their users' perception of the quality of the service they provide, be it academic, pedagogical or administrative [3], [4]. The modeling of user behavior is one of the techniques most used by organizations, with the development of personalized content according to the level of user-platform interaction as its main motivation [5], [6].

In this educational field, the objective is to facilitate learning activities for the user, access to information in an agile way and the management of the required resources [7]. Improving the levels of 
personalization of the content associated with an information system generates a positive perception for the user, making the performance of the services provided more efficient [8], [9]. At present, the development of information technologies allows to store and manage large amounts of data, this applied in virtual education environments generates the possibility of personalizing the content presented to users, through classification models [10], [11].

The classification models are supported by the definition of a priori groups so that through a machine learning model and with known information inputs, the unit of study can be classified into a specific group, in this technique dependent variables are defined and independent [12], [13]. Automatic learning or machine learning, belongs to a branch of artificial intelligence that is based on algorithms that allow modifying the behavior of data based on experience or knowledge acquired autonomously, in order to facilitate the extraction of users. of relevant information [14], [15]. This technique groups together a wide range of algorithms focused on solving various problems, such as: selection of characteristics, classification, grouping or imputation of data, among others [16], [17]. Likewise, the chosen algorithm depends on the type of information analyzed, this allows obtaining higher quality information and improving processing times [18], [19].

Among the algorithms most used in automatic learning for classification models, is the K-nearest neighbors (K-NN), given its simplicity and efficiency to detect and classify elements in categories [20], [21]. This supervised learning algorithm is made up of several descriptive attributes and a single objective attribute (also called class) [22]. The parameter $\mathrm{k}$ in K-NN refers to the number of neighbors with which the belonging to a category is defined, this parameter is usually determined empirically, depending on the problem it is tested with different values of $\mathrm{K}$, choosing the parameter with the best performance in precision [23], [24]. Given what has been described, the present study aims to analyze and discuss the metrics of the predictive model obtained through the supervised learning algorithm K-NN, also known as medium K-NN, applied to the data of the perception of the quality of the virtual administrative service by engineering students, for which the indicators of accuracy, precision, sensitivity and specificity will be analyzed, based on obtaining the confusion matrix and the receiver operational characteristic (ROC) curve, the purpose of this analysis is to provide information quality and relevant to university managers to improve decision-making.

\section{RESEARCH METHOD}

The level of research, according to the degree of measurement and analysis of the information is descriptive, it is based on analyzing and discussing the metrics of the predictive model obtained through the medium K-NN supervised learning algorithm, applied to the data of the perception of the quality of the virtual administrative service by engineering students. Thus, the methodology is based on the construction of a predictive model through the execution of MATLAB software. Figure 1 shows the proposed methodology according to the supervised learning algorithm medium K-NN.

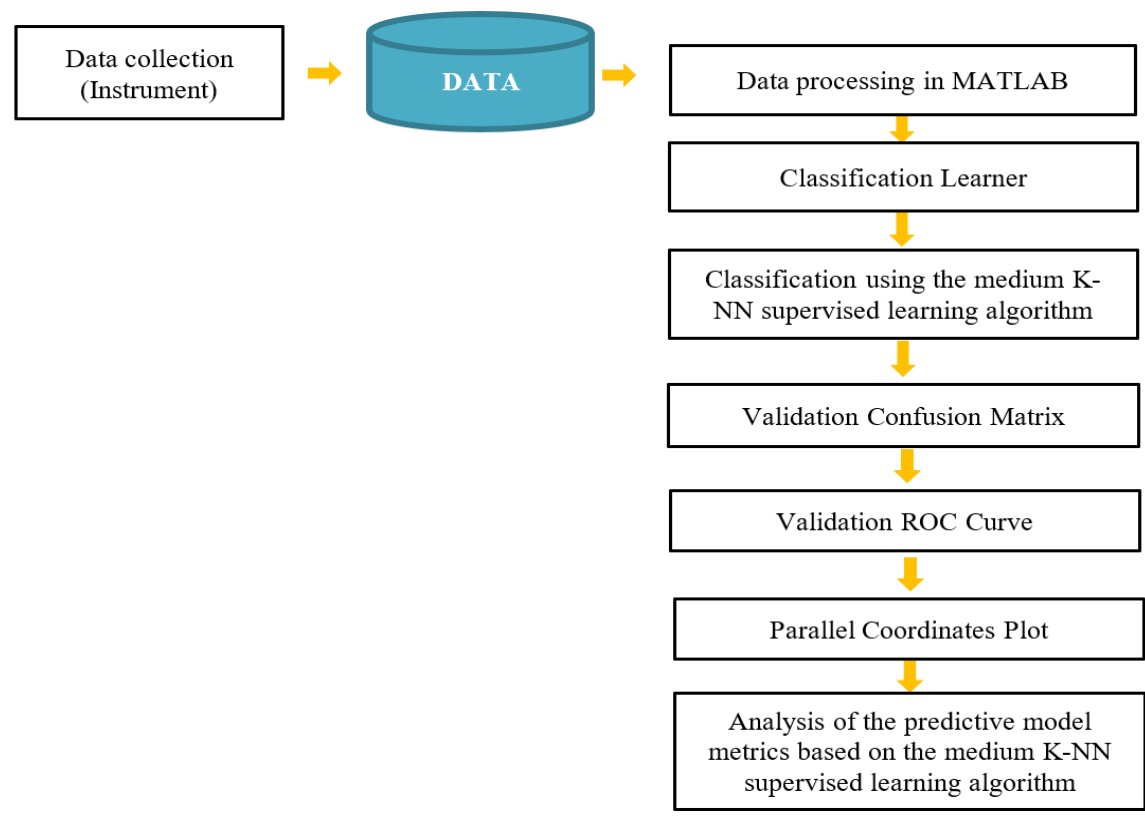

Figure 1. Methodology of the proposed medium K-NN algorithm 
The data referring to the perception of the quality of the virtual administrative service were collected by means of the survey technique, and the collection instrument is the questionnaire, with responses on a Likert scale ranging from 1 to 4, which represent the levels from dissatisfied to very satisfied, these levels of satisfaction in the analysis will be represented as the classes of the model. The survey was carried out virtually due to the context of the health emergency declared by Covid-19, and was applied to all 651 students from the seventh to the tenth cycle, belonging to professional engineering schools, this criterion is part of a regulation established and approved by the university. As part of the methodology, the data collected is validated through Cronbach's Alpha coefficient using the SPSS software; once this analysis has been carried out, it is observed in Table 1 that the values obtained show a high homogeneity and equivalence of the response of all the indicators, since "values greater than 0.9 indicate a great consistency of the elements of the scale" [25]. Likewise, Table 1 shows the indicators called predictors of the quality of the administrative service. Based on these results, the analysis can be continued.

Table 1. Value of Alpha Cronbach's

\begin{tabular}{clc}
\hline Code & \multicolumn{1}{c}{ Indicators } & Alpha Cronbach's \\
\hline I1 & Efficient work & 0.944 \\
I2 & Timely attention & 0.941 \\
I3 & Relevant information provided & 0.942 \\
O1 & Quality care & 0.935 \\
\hline
\end{tabular}

\section{RESULTS AND DISCUSSION}

\subsection{Determination of the predictive model}

This is the training stage, where a classification algorithm builds a model by analyzing or learning from a set of training data. Thus, for the determination of the predictive model, the data collected based on the first 3 indicators shown was used. In Table 1, which are called predictors, while the indicator that represents the quality of the virtual administrative service is represented by 01 . Thus, through the MATLAB software and through the Classification Learner tool and Statistics and Machine Learning Toolbox 12.1, the best type of predictive model determined by the validation of the accuracy is identified. The results generated by the Matlab R2021a software are shown in Table 2.

According to Table 2, of all the learning algorithms, the best type of classification model was granted by the supervised learning algorithm K-NN or medium K-NN, with a validation of $85.5 \%$. Regarding the validation percentage in [21] it is pointed out that, by obtaining $77.85 \%$ precision, it can be stated that the $\mathrm{K}-\mathrm{NN}$ algorithm is capable of classifying well the unbalanced data with the most optimal values. As indicated in [26], the results of the classification and forecasting process show good results in terms of precision when using the medium K-NN algorithm, representing benefits in the ease of interpretation and comparability of the results, in this case with $91 \%$ accuracy exceeds $7.1 \%$ linear and quadratic classifier performance. Contextualizing this result in the university environment, for the educational institution it is important to know the different ways in which the student relates to the educational process and much better if it can take actions to facilitate learning and student satisfaction, re-conceptualizing the management virtual environments of the educational service.

Table 2. Results of classification learner

\begin{tabular}{lc}
\hline \multicolumn{1}{c}{ Algorithm } & Accuracy (validation) \\
\hline Medium K-NN & $85.5 \%$ \\
Bilayered neural network & $85.4 \%$ \\
Bagged trees & $85.3 \%$ \\
\hline
\end{tabular}

\subsection{Results of the predictive model metrics}

The confusion matrix is a useful tool to analyze how well a classification model can correctly predict outcomes in a large number of classes [27]. As indicated in [28] the K-NN algorithm requires knowing in advance the value of $\mathrm{k}$ to determine the $\mathrm{K}$ closest neighbors, the confusion matrix being a common procedure to evaluate different K-NN configurations. Described in the previous paragraph, Figure 2 shows the confusion matrix according to the number of observations, this matrix contains information about the predictions made by the classification system and reports the number of false negatives (FNR) and true positives (TPR), which shows the closeness between the levels of satisfaction predicted (predicted class) by the model with respect to its true value (true class). Rajagopal et al. in [19] it is indicated that the TPR defines the number of positive samples correctly classified as positive and the FNR is the number of positive examples incorrectly classified as negative, these 2 configurations plus the positive values predicted (PPV) and false obtained rate (FDR), shown in Figure 3, will define the performance metrics of the predictive model. 
In Figure 2, it is highlighted that of the 4 classes on which the predictive model acted, class 1 shows the highest percentage of sensitivity, this means that the predictive model has the ability to discriminate between a true positive (TP) of a false negative (FN) in this class (satisfaction level: dissatisfied), in this case it is $89.7 \%$; in other words, the model was only $10.3 \%$ confused, a considerably low rate. Although all the levels are high, we can say that the lowest level of sensitivity of the predictive model is shown in class 2 (level of satisfaction: not very satisfied), whose value is $82.2 \%$. Likewise, in Figure 2, it is highlighted that to the right of the confusion matrix the rate of TPR and the rate of FNR are shown for each class. Regarding the validation of true positives and false negatives, Mhaske-Dhamdhere and Vanjale in [29], the medium algorithm K-NN, allowed to know that out of 160 emails from computer engineering students there are true positives of $67 \%$ and $80 \%$, these values are very high, they are due according to users to the quality of the service in terms of email, such as technical parameters and structure.

In Figure 3, a second confusion matrix is shown in which its main diagonal values indicate the precision of the predictive model for each class. Figure 3 shows that the predictive model for class 3 (satisfaction level: satisfied) shows the best sensitivity rate, with a precision rate of $89.9 \%$, thus being the highest among the other classes. While the lowest level of sensitivity of the predictive model is shown in class 3 (level of satisfaction: very satisfied), whose value is $80.2 \%$. Although the predictive model for class 3 (satisfaction level: satisfied) shows a sensitivity rate of $85.6 \%$ (Figure 2), in this case it shows a precision rate of $89.9 \%$, which indicates that the level of dispersion of the data used is very low; answering that if the dispersion is low the precision is high. Likewise, one aspect to highlight is that in the lower part of the confusion matrix of Figure 3, it should be noted that the PPV and the FDR are shown for each class.

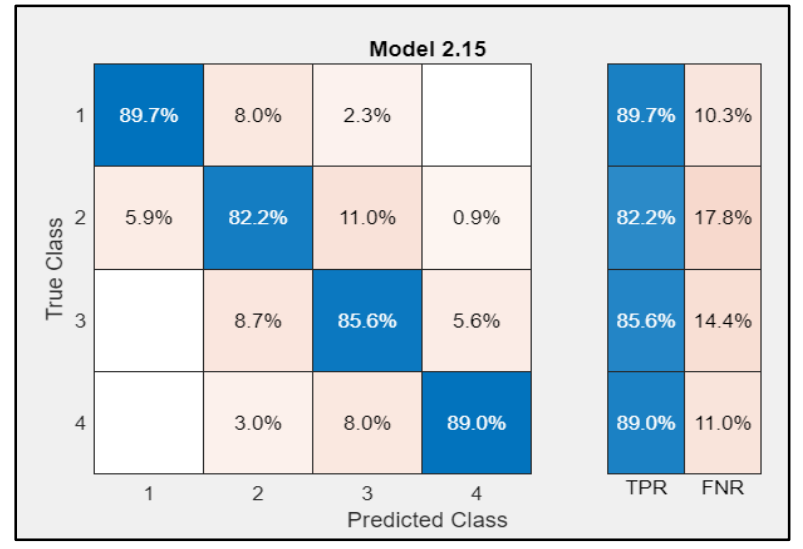

Figure 2. Confusion matrix based on PPV and FDR rates

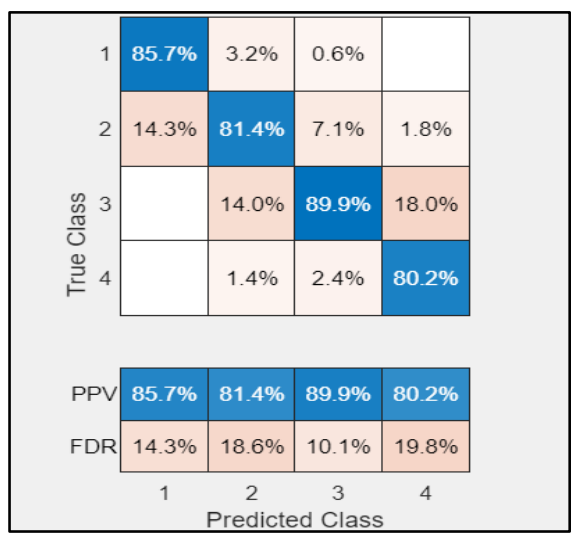

Figure 3. Confusion matrix based on PPV and FDR rates

As noted, the four ranking possibilities of any intrusion detection study determine meaningful performance metrics, such as accuracy (A), precision (P), sensitivity (S), and specificity (R). Table 3 shows the metrics of the predictive model, for each class, which shows that the four metrics show relatively high values in the 4 classes. In general, the accuracy of the predictive model is $85.5 \%$. 
In relation to the sensitivity indicator of the predictive model using the supervised learning algorithm K-NN, Figure 4 shows the response that Matlab provides for each class under study with its corresponding ROC graph, for each class; ROC validation represents the relationship between the sensitivity and specificity indicators; in this case the ROC of class 1 (unsatisfied) is displayed. In Figure 4, the discrimination threshold is shown, which is 0.90 for the rate of true positives and 0.02 for the rate of false positives, showing an AUC of 0.98, being a value almost optimal, very close to one. In the same way, the ROC of class 4 is shown (very satisfied), in Figure 5 the discrimination threshold is shown, which is 0.89 for the rate of true positives and 0.03 for the rate of false positives, evidencing an AUC of 0.96, as indicated, the closest value is 1 , the model is much more optimal.

Table 3. Results of classification learner

\begin{tabular}{ccccc}
\hline \multirow{2}{*}{ Class } & \multicolumn{4}{c}{ Metrics } \\
& Sensitivity & Sensitivity & Accuracy & Precision \\
\hline 1 & $89.66 \%$ & $98.07 \%$ & $97.11 \%$ & $85.71 \%$ \\
2 & $82.19 \%$ & $92.44 \%$ & $89.49 \%$ & $81.45 \%$ \\
3 & $85.63 \%$ & $91.63 \%$ & $88.83 \%$ & $89.94 \%$ \\
4 & $89.00 \%$ & $96.67 \%$ & $95.66 \%$ & $80.18 \%$ \\
\hline
\end{tabular}

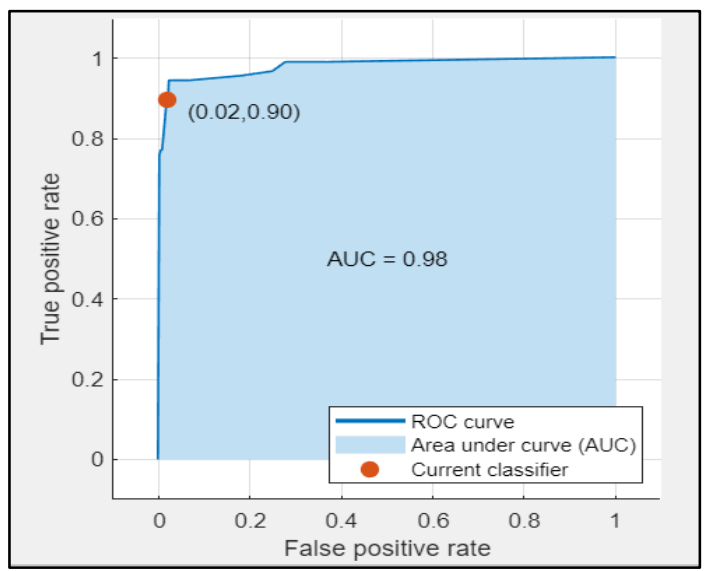

Figure 4. Validation ROC Curve for class 1

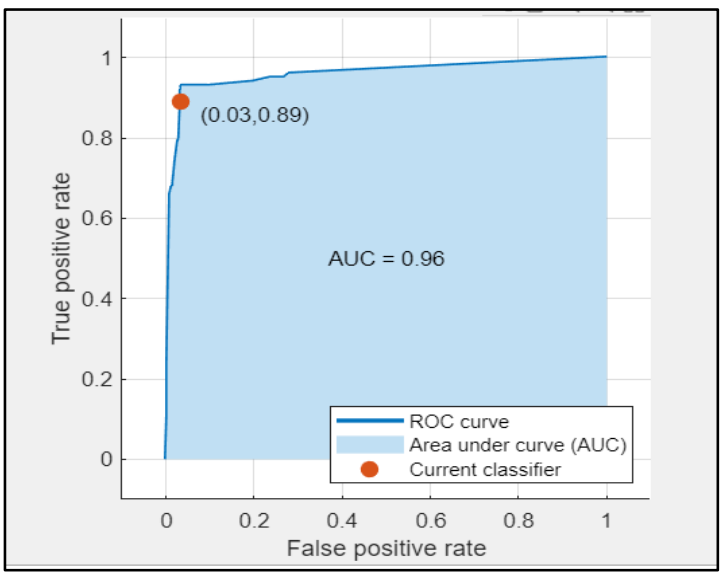

Figure 5. Validation ROC Curve for class 4

Regarding ROC validation, Susheelamma and Ravikuma in [22] it is indicated that there is an improvement in ROC performance, thus reflecting that the proposed learning model improves the score of measure $\mathrm{F}$ by $24.45 \%, 26.65 \%$, and $18.96 \%$ on the existing learning model; with this validation an average improvement of $23.35 \%$ of the existing model is achieved. Finally, after validating the collected data, and proceeding with obtaining the predictive model through the Matlab R2021a software, it was concluded that the average metrics for all its classes were optimal, presenting an accuracy of $92.77 \%$, sensitivity of $86.62 \%$ and specificity of $94.7 \%$; with an overall accuracy of $85.5 \%$. In turn, the highest level of AUC is 0.98 , thus being considered an optimal predictive model.

Regarding what was obtained in [1] it is highlighted that, from the perspective of innovation, the results of these studies achieve great changes, they also allow delegating functions, promoting skills, and encouraging continuous updating; all this from the visionary leadership approach. Likewise, what was obtained in [22] indicates that the proposed model accurately predicts even for the early day (that is, during 0 and 1 days), it also efficiently predicts the days after the end of the course and achieves better results than training with legacy data. As indicated in [30] there is a strong tendency to predict student performance in college. For what research about predicting the behavior of students in the academic environment, it is very interesting, because within an organization the quality of service is essential, since user satisfaction depends on it. The results Ghouch et al. in [31] indicate that the integration of the K-NN algorithm in the educational environment allows searching for students with similar behaviors, which will offer, on the one hand, a learning path adapted to the student's profile, and based on the experiences of others. students with similar behaviors by observing and analyzing their learning footprints, and, on the other hand, overcomes the limitations of the K-NN algorithm in terms of computational time and memory. 


\section{CONCLUSION}

In relation to the results obtained, an optimal predictive model is evidenced, with an accuracy of $85.5 \%$ making use of the supervised learning algorithm K-NN, likewise, the application of the crossvalidation procedure shows optimal results in the experimentation of the K-algorithm. NN finding all the model metrics (sensitivity, specificity, accuracy, and precision) in the 4 classes with relatively high values, said this, the results will allow to establish the grouping of engineering students who can reach a level of satisfaction based on the indicators called predictors, by means of which the authorities will be able to make timely decisions to improve the percentage of satisfied students and reduce the percentage of dissatisfied students in relation to the quality of the virtual administrative service, also these classification techniques allow to extract relevant information from interested parties, from higher quality and even less time. The research also provides added value, since it provides the scientific and academic community with a methodology to classify participating students in virtual environments, identifying the relationship between the predictive elements and the results of the satisfaction of the quality of the administrative service, which is provides in this context virtually.

\section{ACKNOWLEDGEMENTS}

The authors wish to recognize and thank the National Technological University of South Lima for their support of this investigation.

\section{REFERENCES}

[1] O. A. M. Molina, "Visionary Leadership In The Administrative Staff Of The Guapan Educational Unit," Journal of Technology and Science Education, vol. 8, no. 2, pp. 115-125, March. 2018, doi: 10.3926/jotse.413.

[2] S. Abdulla and A. Al-Nassiri, "kEFCM: KNN-Based Dynamic Evolving Fuzzy Clustering Method," International Journal of Advanced Computer Science and Applications (IJACSA), vol. 6, no. 2, pp. 5-13, Feb. 2015, doi: 10.14569/IJACSA.2015.060202.

[3] B. Bahati, U. Fors, P. Hansen, J. Nouri, and E. Mukama, "Measuring Learner Satisfaction with Formative e-Assessment Strategies," International Journal of Emerging Technologies in Learning, vol. 14, no. 7, pp. 246-247, Dec. 2019, doi: 10.3991/ijet.v14i07.9120.

[4] D. Supriadi and U. Sa'ud, "The Effectiveness of Implementing Information and Communication Technology On Student Academic Services (A Case Study in Bandung Institute of Technology for the 2015-2016 Period), International Journal of Education, vol. 9, no. 2, pp. 139-148, Feb. 2017, doi: 10.17509/ije.v9i2.5478.

[5] C. G. Cegielski and A. Jones-Farmer, "Knowledge, skills, and abilities for entry-level business analytics positions: a multimethod study," Decision Sciences Journal of Innovative Education, vol. 14, no. 1, pp. 91-118, Jan. 2016, doi: 10.1111/dsji.12086.

[6] E. Ahumada and J. M. Perusquia, "Business intelligence: strategy for the development of competitiveness in technology-based companies," Accounting and Administration, vol. 61, no. 1, pp. 127-158, Oct. 2016, doi: 10.1016/j.cya.2015.09.006.

[7] Q. M. Shallal, Z. A. Hussien, and A. A. Abbood, "Method to implement K-NN machine learningto classify data privacy in IoT environment," Indonesian Journal of Electrical Engineering and Computer Science (IJEECS), vol. 20, no. 2, pp. 985-990, Nov. 2020, doi: 10.11591/ijeecs.v20.i2.pp985-990.

[8] R. Olu-Ajayi, "An Investigation into the Suitability of K-Nearest Neighbour (K-NN) for Software Effort Estimation," International Journal of Advanced Computer Science and Applications(IJACSA), vol. 8, no. 6, pp. 227-233, Jun. 2018, doi: 10.14569/IJACSA.2017.080628.

[9] P. Mesároš, S. Carnicky, T. Mandičák, and M. Habinakova, "Business Intelligence impact on corporate performance in Slovak enterprises - a case study," Journal of Systems Integration, vol. 7, no. 4, pp. 9-18, Apr. 2016, doi: 10.20470/jsi.v7i4.267.

[10] K. C. Waghmare and B. A. Sonkamble, "Modified K-nearest Neighbor Algorithm with Variant K Values," International Journal of Advanced Computer Science and Applications (IJACSA), vol. 11, no, 10, pp. 220-224, Oct. 2020, doi: 10.14569/IJACSA.2020.0111029.

[11] D. Buenaño-Fernández, D. Gil, and S. Luján-Mora, “Application of Machine Learning in Predicting Performance for Computer Engineering Students: A Case Study,” Sustainability, vol. 11, no. 10, p. 2833, May 2019, doi: 10.3390/su11102833.

[12] V. Pedrero, K. Reynaldos-Grandón, J. Ureta-Achurra, and E. Cortez-Pinto, "Overview of machine learning and its application in the management of emergency services," Medical journal of Chile, vol. 149, no. 2, pp. 248-254, Feb. 2021, doi: 10.4067/s003498872021000200248 .

[13] T. Bailetti, M. Gad, and A. Shah, "Intrusion Learning: An Overview of an Emergent Discipline," Technology Innovation Management Review, vol. 6, no. 2, pp. 15-20, Feb. 2016.

[14] W. Cherif, A. Madani, and M. Kissi, "A combination of low-level light stemming and support vector machines for the classification of Arabic opinions," 2016 11th Int. Conf. Intel. Sys.: Theo. App., 2016, pp. 1-5, doi: 10.1109/SITA.2016.7772290.

[15] S. Damuluri, K. Islam, P. Ahmadi, and N. S. Qureshi's, "Analyzing Navigational Data and Predicting Student Grades Using Support Vector Machine,” Emerging Science Journal, vol. 4, no. 4, pp. 243-252, Aug. 2020, doi: 10.28991/esj-2020-01227.

[16] C. Gonzales, E. Elhariri, N. El-Bendary, A. Fernández, and R. P. Díaz, "Machine learning based classification approach for predicting students performance in blended learning," Adv. Intell. Syst. Comput, vol. 407, pp. 47-56, Nov. 2016, doi: 10.1007/9783-319-26690-9_5.

[17] H. Tien-Chi, L. Rynson, H. Yueh-Min, S. Marc, and Y. Chun-Hung, "The jacobian matrix-based learning machine in student. Lect. Notes Comput," International Symposium on Emerging Technologies for Education, 2017, doi: 10.1007/978-3-319-71084-6_55.

[18] S. B. Kotsiantis, I. Zaharakis, and P. Pintelas, "Supervised machine learning: A review of classification techniques," Emerging artificial intelligence applications in computer engineering, vol. 160, no. 1, pp. 3-24, Jun. 2007, doi: 10.5555/1566770.1566773.

[19] S. Rajagopal, K. S. Hareesha, and P. Kundapur, "Performance analysis of binary and multiclass models using azure machine learning," International Journal of Electrical and Computer Engineering (IJECE), vol. 10, no. 1, pp. 978-986, Feb. 2020, doi: 10.11591/ijece.v10i1.pp978-986. 
[20] A. K. Jain, "Data clustering: 50 years beyond K-means," Pattern Recognition Letters, vol. 31, no. 8, pp. 651-666, Jun. 2010, doi: 10.1016/j.patrec.2009.09.011.

[21] A. Aprilia, M. A. Fauzi and M. T. Furqon "Neighbor Weighted K-Nearest Neighbor for Sambat Online Classification," Indonesian Journal of Electrical Engineering and Computer Science, vol. 12, no. 1, pp. 155-160, Oct. 2018, doi: 10.11591/ijeecs.v12.i1.pp155-160.

[22] K. H. Susheelamma and K. M. Ravikuma, "Student risk identification learning model using machine learning approach," International Journal of Electrical and Computer Engineering (IJECE), vol. 9, no. 5, pp. 3872-3877, Oct. 2019, doi: 10.11591/ijece.v9i5.pp3872-3879.

[23] I. Hadi and A. Mahdi, "Generating images of partial face using landmark based K-nearest neighbor," Indonesian Journal of Electrical Engineering and Computer Science, vol. 17, no. 1, pp. 420-428, Jan. 2020, doi: 10.11591/ijeecs.v17.i1.pp420-428.

[24] N. M. N. Mathivanan, N. A. Md.Ghani, and R. M. Janor, "A comparative study on dimensionality reduction between principal component analysis and k-means clustering," Indonesian Journal of Electrical Engineering and Computer Science, vol. 16, no. 2, pp. 752-758, Nov. 2019, doi: 10.11591/ijeecs.v16.i2.pp752-758.

[25] J. Mutahi, A. Kinai, N. Bore, A. Diriye, and K. Weldemariam, "Studying engagement and performance with learning technology in an African classroom," in Proceedings of Seventh International Learning Analytics \& Knowledge Conference, 2017, pp. 148152, doi: $10.1145 / 3027385.3027395$

[26] E. J. De-La-Hoz and T. J. Fontalvo, "Methodology of Machine Learning for the classification and Prediction of users in Virtual Education Environments," Technological information, vol. 30, no. 1, pp. 247-254, Feb. 2019, doi: 10.4067/S071807642019000100247.

[27] A. D. Poernomo and S. Suharjito, "Indonesian online travel agent sentiment analysis using machine learning methods," Indonesian Journal of Electrical Engineering and Computer Science, vol. 14, no. 1, pp. 113-117, Apr. 2019, doi: 10.11591/ijeecs.v14.i1.pp113-117.

[28] H. R. G. Diez, G. Santos, F. Campos, and C. Morell, "Evaluation of KNN-SP algorithm for multi-target prediction problems in Spanish: Evaluación del algoritmo KNN-SP para problemas de predicción con salidas compuestas," Cuban Journal of Computer Science, vol. 10, no. 3, pp. 119-129, 2016.

[29] V. Mhaske-Dhamdhere and S. Vanjale, "A Novel Approach for Phishing Emails Real Time Classification Using K-Means Algorithm," International Journal of Electrical and Computer Engineering (IJECE), vol. 8, no. 6, pp. 5326-5332, Dec. 2018, doi: 10.11591/ijece.v8i6.pp5326-5332.

[30] J. L. Rastrollo-Guerrero, J. A. Gómez-Pulido, and A. Durán-Domínguez, “Analyzing and Predicting Students' Performance by Means of Machine Learning: A Review," Applied Sciences, vol. 10, no. 3, p. 1042, Feb. 2020, doi: 10.3390/app10031042.

[31] E. G. Nihad, E.-N. El Mokhtar, Z. Abdelhamid, and A. A. Mohammed, "Hybrid approach of the fuzzy C-means and the K-nearest neighbors methods during the retrieve phase of dynamic case based reasoning for personalized follow-up of learners in real time," International Journal of Electrical and Computer Engineering (IJECE), vol. 9, no. 6, pp. 4939-4950, Dec. 2019, doi: 10.11591/ijece.v9i6.pp4939-4950.

\section{BIOGRAPHIES OF AUTHORS}
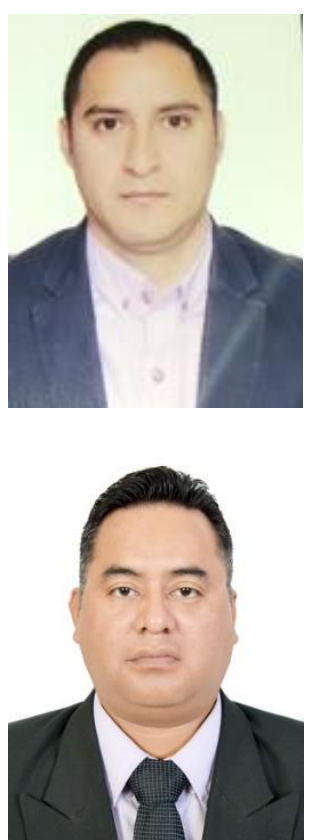

Omar Freddy Chamorro Atalaya (iD $8 \mathrm{SC}$ P is an electronic engineer, graduated from the National University of Callao (UNAC), with a Master's degree in Systems Engineering and a doctoral student at the Faculty of Administrative Sciences at UNAC. Researcher recognized by CONCYTEC (National Council for Science, Technology and Technological Innovation). Research professor at the Universidad Nacional Tecnológica de Lima Sur (UNTELS), in the Associate category, he teaches courses on automatic process control and industrial automation, and design of control panels and electrical control. He is the author of scientific articles indexed to Scopus and WoS. He is a reviewer for scientific articles for journals indexed to Scopus. He is a speaker at scientific conferences, in areas such as Data Science, Machine Learning, Natural Language Processing, and Sentiment Analysis. He can be contacted at email: ochamorroa@untels.edu.pe

Guillermo Morales Romero (D) SC SC P External Evaluator in University Higher Education at SINEACE, Doctor in Educational Sciences, Master in Systems Engineering, Master in Public Management, Master in Educational Management, Bachelor of Mathematics and Computer Science, Lawyer with specialist in computer auditing, computer security, X cycle Systems Engineering of UNFV, Professional with 24 years of experience in University Teaching in the careers of Engineering, Computer Science, Law, Mathematics Education, Statistics. Teacher in the different Postgraduate Schools of Public and Private Universities of the Country. Author of scientific articles in journals indexed in databases: Scopus, Wos, Latindex and Others. Organizer of National and International Events, National and International Speaker. Double Blind Pair Reviewer of different national and international scientific journals. Member of the Scientific Committee of the Scientific Journal: Ciencia \& Sociedad Autonomous University Tomás Frías de Bolivia; Member of the Scientific Committee of the Multidisciplinary Magazine Ciencia Latina. Mexico. Member of the Lima Chamber of Commerce and the Lima Bar Association. Former specialist in the AGEBAT Area of UGEL 05 - MINEDU. He can be contacted at email: gmorales@ une.edu.pe 

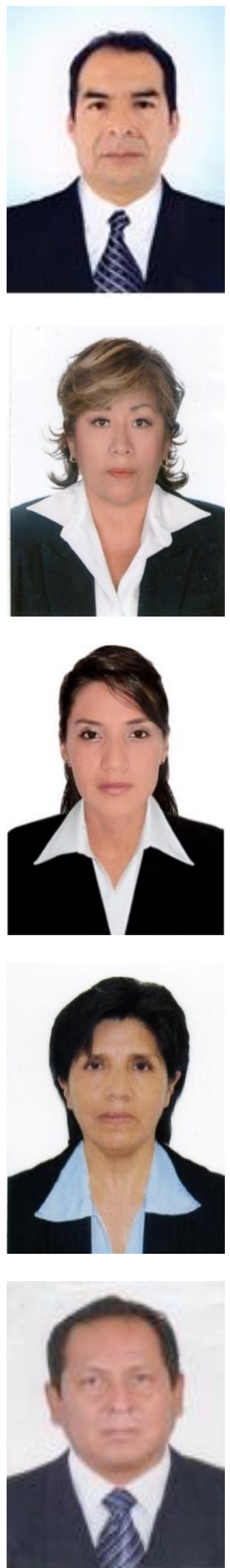

Adrián Quispe Andía (D) SC S D Doctor in Educational Sciences, with a Master's Degree in University Didactics, a Bachelor of Computer Science from the Universidad Nacional Mayor de San Marcos and a Bachelor of Education specializing in mathematics from the Enrique Guzmán y Valle University, with completed studies in Systems Engineering at the National University of San Marcos. With more than 25 years of experience as an undergraduate and graduate university teacher in different public and private institutions. My line of research is oriented to University Higher Education, author of books on statistics applied to scientific research at national and international level, I carry out research projects with competitive funds and participate in national and international conferences as a speaker, in addition to publishing articles scientists in international journals and indexed in high-impact repositories such as Scopus. He can be contacted at email: aquispe@une.edu.pe.

Beatriz Caycho Salas (D) SC P Doctor in educational sciences, Master in Educational Management, graduate of master's degree in administration, Bachelor of Administration, Teacher of Primary Education, graduate of the professional education career with the mention of social sciences and educational administration, professional with 27 years of experience in University Teaching in the careers of administration, international business and undergraduate education. Postgraduate teaching experience: Public Management. Co-author of university texts. She can be contacted at email: bcaycho@une.edu.pe

Elizabeth Katerin Auqui Ramos (D) 8d SC P Magister in the mention of University Teaching, with completed doctorate studies in the mention of Educational Sciences, studies in Industrial Engineering. Proficiency in English at an intermediate level, Italian and Quechua at a basic level, graduates in didactics of mathematics and pedagogical management and innovation for educational quality and competitiveness, with 11 years of experience in teaching at higher and university levels. She can be contacted at email: eauqui@une.edu.pe

Primitiva Ramos Salazar (ID) SC P with completed doctoral studies in educational sciences, Master's degree in educational sciences, completed Master's studies in administration, Bachelor's degree in textile technology, with 23 years of experience in teaching technical-productive education and 7 years in university teaching. She can be contacted at email: pramos@une.edu.pe

Carlos Palacios Huaraca (D) 8d SC $\mathrm{P}$ Doctor of Educational Sciences, Master of Mathematical Education, Bachelor of Mathematics and Computer Science, second specialty in Mathematics Didactics with 11 years of experience in University Teaching in Engineering and Education careers. He can be contacted at email: c18125@utp.edu.pe 